\title{
Experimental Demonstration of Perching by an Articulated Wing MAV
}

\author{
Aditya A. Paranjape*, Joseph Kim† Nihar Gandhił and Soon-Jo Chung§
}

This paper presents an experimental demonstration of perching by a micro aerial vehicle (MAV) equipped with articulated wings. A novel feature of the MAV considered in this paper is that wing dihedral, controlled independently on both wings, is used for yaw stability and control as well as for maintaining the flight path angle. Yaw stability and control are essential for perching in tightly constrained places. The experiments described in this paper were conducted indoors and flight parameters are measured using the VICON motion capture system.

\section{Nomenclature}

$\begin{array}{cl}C_{L}, C_{D} & \text { coefficients of lift and drag } \\ C_{m, a c} & \text { coefficient of pitching moment about the aerodynamic center } \\ N & \text { yawing moment } \\ \alpha, \beta & \text { angle of attack, sideslip } \\ \psi, \theta, \phi & \text { Euler angles } \\ \gamma & \text { flight path angle } \\ p, q, r & \text { body axis roll, pitch and yaw rates } \\ \delta_{L}, \delta_{R} & \text { dihedral angle of left and right wing } \\ \delta_{a}, \delta_{s} & \text { asymmetric dihedral angle }\left(\delta_{R}=-\delta_{L}=-\delta_{a}\right) \text { and symmetric dihedral angle } \\ \delta_{e} & \text { elevator or horizontal tail deflection }\end{array}$

\section{Introduction}

There is a growing interest in the aerospace community in the development of Micro Aerial Vehicles (MAV) to learn and mimic avian flight. MAVs fly in low Reynold number regimes of $10^{3}$ to $10^{5}$, which corresponds to that of small birds. ${ }^{1}$ MAVs with wings equipped with multiple degrees-of-freedom such as flapping, wing twist and sweep provide greater manueverability than conventional fixed-wing aircraft. These MAVs can be used for intelligence gathering, surveillence, and reconnaissance missions in tightly constrained spaces such as forests and urban areas. Advances in actuators and control systems have led to development and analysis of articulated MAVs inspired by birds. ${ }^{2}$ Birds achieve remarkable stability and perform agile manuevers using their wings very effectively. ${ }^{3}$ One of the goals of reverse-engineering avian flight is to learn more about the various aspects of avian flight such as stability, maneuverability and control from the dynamics of MAV.

One of the bioinspired manuevers which is presently of interest is perching. Perching can be described as a high angle-of-attack pull-up with high lift and a large drag. The large lift and drag forces cause the MAV to climb and lose speed significantly. A planted landing can be achieved in the process. ${ }^{4,5}$

\footnotetext{
*Doctoral student, Department of Aerospace Engineering, University of Illinois at Urbana-Champaign, Urbana, IL 61801. Email: paranja2@illinois.edu. Student Member, AIAA.

${ }^{\dagger}$ Undergraduate Research Assistant, Department of Aerospace Engineering, UIUC. Email: kim323@illinois.edu

${ }^{\ddagger}$ Undergraduate Research Assistant, Department of Aerospace Engineering, UIUC. Email: gandhi9@illinois.edu. Student Member, AIAA.

$\S$ Assistant Professor, Department of Aerospace Engineering, UIUC. Email: sjchung@illinois.edu. Senior Member, AIAA.
} 
Birds successfully perch on a variety of structures such as building ledges, power lines, cliff side, and tree branches. Such perching capability in MAVs can significantly reduce the landing distance. However, perching requires the ability to maintain trajectory very accurately. Furthermore, a typical perching maneuver would not last more than a few seconds. Because of its duration and highly unsteady flight profile, perching is an important agility metric for MAVs. The unsteady flight profile makes control design for perching a challenging problem.

The aerodynamics of perching has been explored for conventional, fixed-wing aircraft by Crowther. ${ }^{6}$ Controllability issues were noted during deep stall, and a large undershoot of the trajectory onto the landing site was discovered. Wickenheiser and Garcia demonstrated perching manuever with controlled wing twist and variable tail incidence. ${ }^{5,7}$ Reich et al. ${ }^{8}$ experimentally studied the aerodynamic performance of a wing of variable incidence for perching. Roberts et al. ${ }^{9}$ examined the perching problem from controllability aspects. Desbiens and Cutkosky ${ }^{10}$ examined the problem of perching on vertical surfaces.

The primary goal of the present paper is to demonstrate the capabilities and the limitations of using the wing dihedral for longitudinal as well as lateral-directional control. Lateral-directional control is usually ignored in the context of perching, but since the aircraft considered in this paper is susceptible to yaw instabilities, yaw control is an essential part of the maneuver. The control challenge is enhanced by the design of the MAV considered in this paper. The MAV lacks a vertical tail, but features a novel yaw control mechanism utilising the dihedral of both wings independently of each other. ${ }^{3}$ Using wing dihedral for yaw control is highly unconventional, but in flapping wing aircraft, the ability to control wing dihedral exists by virtue of design. In such cases, a vertical tail becomes redundant, even expensive, and wing dihedral emerges as a natural candidate actuator for yaw control during gliding flight phases.

To effect the most rapid decrease in kinetic energy while perching, flying in a stalled flow regime is desirable along with minimization of the stall speed. However, stability problems may arise due to unsteady forces from the separated wake, where the effectiveness of control surfaces dramatically diminishes. ${ }^{7}$ Interestingly, in such circumstances, wing dihedral is much more effective at lateral-directional control than any control surface in the wing wake.

The objective of the present paper is to show that MAVs can perform a satisfactory perching manuever using a combination of variable wing dihedral and elevator. Additionally, asymmetric dihedral deflection of the wings can reject small pertubations and maintain lateral stability as long as the roll rate does not build up significantly. All experiments were performed indoors. The VICON motion capture system, using 16 2 Mega-pixel (MP) cameras, was used extract aircraft position and attitude accurately. VICON has been employed fruitfully by researchers elsewhere as well to study MAV flight (see Ref. [11,12], for example). A real-time controller which uses VICON data as feedback is implemented.

The rest of the paper is organized as follows. The theoretical underpinnings of this paper have been reviewed briefly in Sec. II. The design of the control law is described in Sec. III. The experimental aircraft as well as the experimental set up are described in Sec. IV. Experimental results have been presented in Sec. V, while Sec. VI concludes the paper.

\section{Summary of Flight Mechanics with Articulated Wings}

The work presented in this paper is based on Ref. [3], where the concept of dihedral-based control for MAVs was described and analysed extensively. A few important observations have been recapitulated in this section. Figure 1 illustrates the physics underlying the use of wing dihedral as a control. Increasing the wing dihedral reduces the force acting in the body $z$-direction, and generates a side force. The reduced $z$-force affects the aircraft flight path angle and angle of attack, and hence the flight speed. On the other hand, the side force can be used for providing the centripetal force for turning, and as a source of the yawing moment. In particular, if the CG is located behind the line of action of the side force, then a positive side force produces a positive yawing moment and vice-versa. It follows that a positive rolling moment (wherein the lift on the left wing is higher than the right wing) is accompanied by a positive yawing moment if the wings have a positive dihedral deflection. Consequently, the adverse yaw produced due to rolling is reduced.

The yaw control effectiveness of the wing dihedral (measured in terms of the yawing moment produced per unit deflection) is tempered by the negative pitching moment produced by wings with a positive camber. The yaw control effectiveness, $N_{\delta a}$, is approximated as follows: ${ }^{3}$

$$
N_{\delta_{a}} \approx \frac{1}{2 I_{z}} \rho V^{2} S_{\text {out }} c\left(\frac{C_{L_{\alpha}} \alpha}{3}-C_{m, a c}\right)
$$




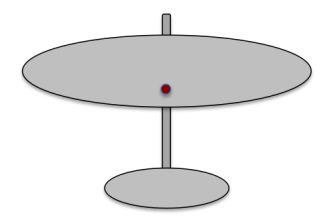

Wing AC ahead of CG

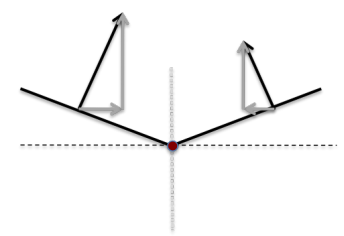

Excess lift on one wing produces side force and yawing moment

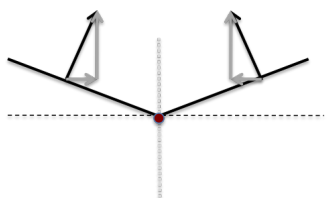

Reduce net lift on aircraft without altering drag

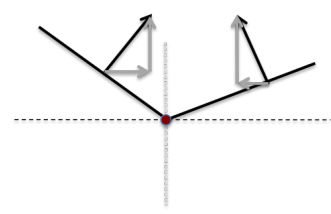

Larger dihedral on one side produces side force and yawing moment

Figure 1. The physics underlying the use of wing dihedral for longitudinal and yaw control

where $S_{\text {out }}$ is the combined area of the outboard sections of the two wings and $I_{z}$ is the aircraft moment of inertia about the $z$ axis. The effectiveness can be negative at low angles of attack for wings with positive camber $\left(C_{m, a c}<0\right)$. Thereafter, for a range of angles of attack, control effectiveness is sensitive to the angular rates before it becomes positive uniformly across the routinely flown flight envelope. This phenomenon has been illustrated in Fig. 2, borrowed from Ref. [3]. The angle of attack, at which the effectiveness ceases to be negative, increases with increasing wing camber. The reader may be tempted to assume that the issue of negative control effectiveness only affects controllability and can be dealt with as such. However, it can have a significant impact on the turning performance of the aircraft. At low angles of attack, for example, an entry into right turns requires that the left wing dihedral be larger than the right wing dihedral to generate the required positive side force. This configuration, however, produces a negative yawing moment which inhibits the turn. The only way to address this problem effectively is to use wing twist or ailerons. At the same time, it must be noted that controlling the wing dihedral deflections is sufficient to ensure stabilization and yaw rate regulation.

Finally, although this may be obvious to most readers, it is worth recalling that the absence of a vertical tail renders the lateral-directional dynamics unstable. Open loop gliding tests were performed on the aircraft used for experiments described in this paper, as well as on a similar aircraft described in Ref. [3]. The tests showed that, depending on the initial condition, the aircraft could simply veer off a straight path gently but rapidly or even enter into a steep spin. In either case, the divergence was rapid, with a time constant of approximately $0.2 \mathrm{~s}$. The key difference between the two outcomes is the build-up of roll rate. The dihedralbased mechanism described here can bring about rapid changes in the yaw rate, but it is significantly less effective at regulating the roll rate.

\section{Control Law Design}

Control law design for the MAV has been described in this section. The control law has a two-tier hierarchical structure based on time-scale separation ${ }^{19}$ which occurs naturally between the fast rotational dynamics and the slow translational dynamics:

- The innermost loop commands the elevator and the asymmetric components of the wing dihedral.

- The outer loop commands the angle of attack and turn rate to be tracked by the inner loop based on flight speed and turn rate. The turn rate and the flight path angle are computed based on position measurements.

A schematic of the controller has been shown in Fig. 3 . 


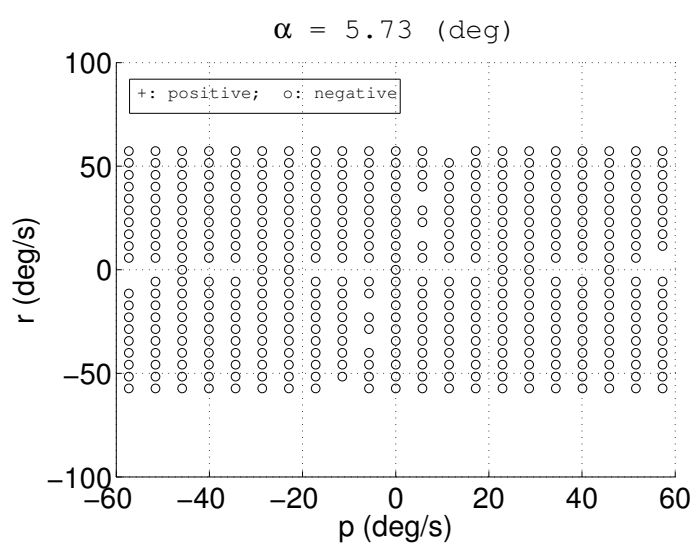

(a) Control effectivness at $\alpha=5.73 \mathrm{deg}$

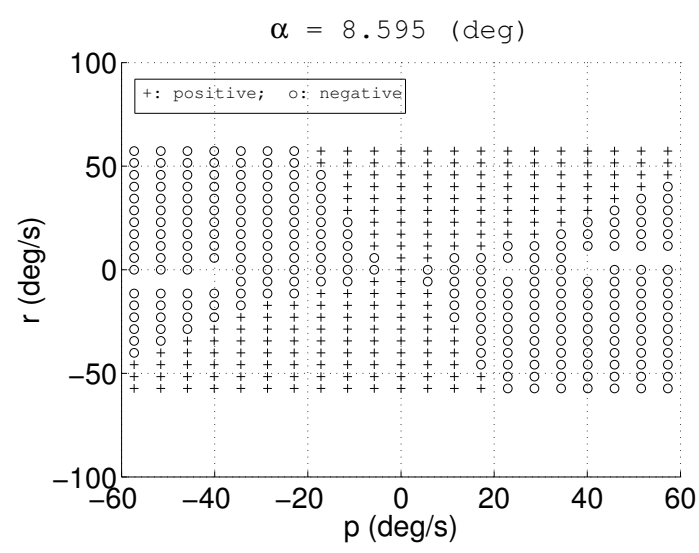

(b) Control effectivness at $\alpha=8.595 \mathrm{deg}$

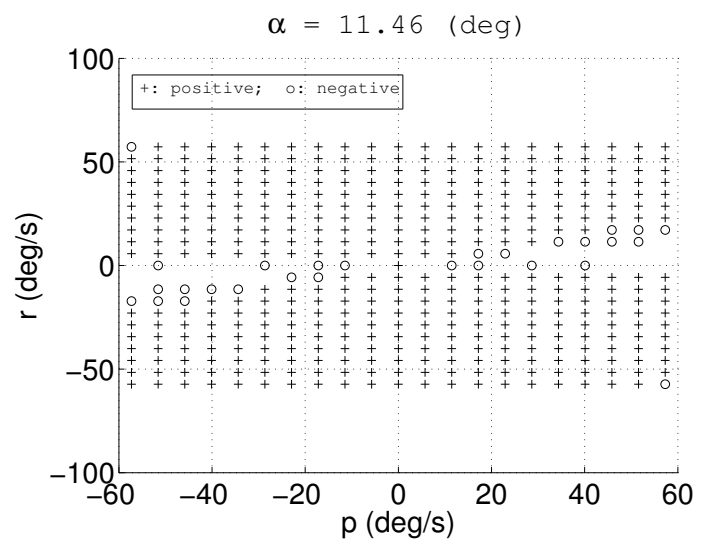

(c) Control effectivness at $\alpha=11.46 \mathrm{deg}$

Figure 2. Plots showing the sign of the control effectiveness, sign $\left(\frac{\Delta N}{\Delta\left(\delta_{L}-\delta_{R}\right)}\right)$, as a function of the angle of attack, roll rate and yaw rate [3].

\section{A. Angle of Attack Control}

The stability of the longitudinal dynamics depends on the CG location. Two longitudinal controllers were designed: one for the configuration with the vertical tail where the $\mathrm{CG}$ was placed around the quarter-chord point of the wing (hereafter referred to as Configuration A), and another for the configuration without a vertical tail where the $\mathrm{CG}$ was placed between $0.25 c$ and $0.3 c$ behind the wing $\mathrm{AC}$ (hereafter called Configuration B). Here, $c$ denotes the wing root chord length.

The longitudinal dynamics of Configuration A were seen during experiments to be stable across the angle of attack envelope, as a consequence of a favorable CG location, while the lateral dynamics showed a divergent unstable yaw mode. The angle of attack is controlled using a simple PID scheme which ensures satisfactory tracking and retains an ease of implementation on the hardware.

Let $e_{\alpha}(t)=\alpha_{c}(t)-\alpha(t)$, where $\alpha_{c}(t)$ is the commanded angle of attack. A gain-scheduled PI controller commands the elevator deflection in configuration $\mathrm{A}$ :

$$
\delta_{e}(t)=k_{p} e_{\alpha}+k_{i} \int_{0}^{t} e_{\alpha} d t, \text { where } k_{p}=k_{i}=-0.45+20(\alpha-0.18)^{2}
$$

The control gains were chosen using the Ziegler-Nichols method after locating the value of $k_{p}$, with $k_{i}=0$, which marked the onset of oscillatory instability in $\alpha$. 


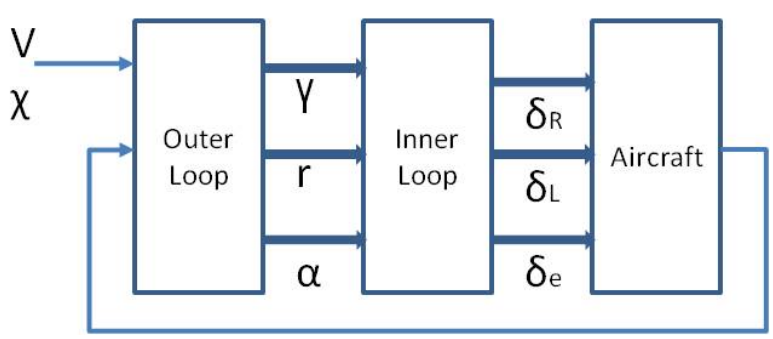

Figure 3. Schematic of the controller, where $\chi$ denotes the aircraft heading.

The longitudinal dynamics of Configuration B are stable, but poorly damped for $\alpha>8$ deg. Around $\alpha=15 \mathrm{deg}$, the elevator effectiveness saturates and higher angles of attack are unattainable under routine flight conditions. The open loop response was measured to have a time period of $1 \mathrm{~s}$. The observed reduction in the amplitude of oscillations was used to approximate the damping coefficient to 0.046. The open loop dynamics can be written in the form

$$
\ddot{\alpha}+0.62 \dot{\alpha}+40 \alpha=-40 \delta_{e}+5.6
$$

Therefore, a derivative-integral controller is designed for Configuration B:

$$
\delta_{e}(t)=0.14-\alpha_{c}+k_{d} \dot{e}+k_{i} \int_{0}^{t} e_{\alpha} d t
$$

where the offset of $0.14 \mathrm{rad}$ is added based on the measured $\delta_{e}-\alpha$ trims. The gain $k_{i}$ is similar to that for Configuration A, while $k_{d}=0.217$ is chosen so that the damping coefficient is approximately equal to 0.7 .

\section{B. Yaw Control}

Yaw control has been often neglected in the literature on perching, mostly because the aircraft possessed the traditional roll and yaw surfaces (or their equivalents). On the other hand, yaw control is an important concern for aircraft, such as the one considered in this paper, which lack a roll control surface and use a highly unconventional yaw control mechanism. Two different yaw controllers are needed for Configurations A and B because the wing dihedral plays a separate role in each configuration. Moreover, although both configurations are laterally unstable, the nature of the instability is different. In Configuration A, the role of the wing dihedral is to primarily provide the side force required to sustain a turn. The yaw moment required for trimming comes from the wing dihedral as well as the vertical tail. Furthermore, since the vertical tail is not actuated, the dihedral angles need to be controlled for different maneuvers such as entering or recovering from a turn.

The asymmetric component of the wing dihedral angles, $\delta_{a}$ (not the aileron deflection), is commanded by a PI controller. Let $e_{r}(t)=r_{c}(t)-r(t)$, where $r_{c}(t)$ is the commanded yaw rate. The anti-symmetric dihedral deflection commanded by the controller is given by

$$
\delta_{a}(t)=1 e_{r}(t)+0.5 \int_{0}^{t} e_{r}(t) d t
$$

Unlike Configuration A, Configuration B is seen to be highly unstable in the open loop. Figure 4 is an illustration of the nature of lateral stability in tailless aircraft for various values of (symmetric) wing dihedral angle. Note that the lateral-directional dynamics are primarily underdamped, which mandates the use of a derivative controller (unlike the PI which sufficed for Configuration A). form

Based on experimental observations, it was estimated that the open loop yaw-rate dynamics are of the

$$
\ddot{r}+2 \xi \omega \dot{r}+\omega^{2} r=N_{\delta a} \delta_{a}, \quad \xi \approx-0.1, \quad \omega \approx 2 \pi
$$

for $\alpha<8$ deg. Thereafter, the yaw dynamics are unstable and oscillatory in nature. Recall the approximation for $N_{\delta a}$ :

$$
N_{\delta_{a}} \approx \frac{1}{2 I_{z}} \rho V^{2} S_{\text {out }} c\left(\frac{C_{L_{\alpha}} \alpha}{3}-C_{m, a c}\right)
$$




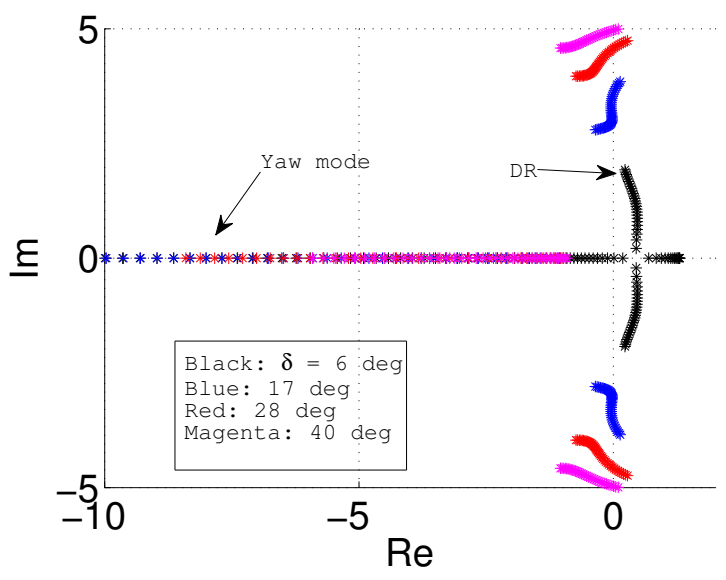

Figure 4. Eigenvalues of the linearized lateral dynamics of a tailless aircraft, from Ref. [3], for different values of the dihedral deflection.

where $S_{\text {out }}$ is the combined area of the outboard sections of the two wings and $I_{z}$ is the aircraft moment of inertia about the $z$ axis. Substituting the estimates for the geometric and aerodynamic terms, it follows that

$$
-2<N_{\delta a}<-1.2, \quad \alpha<8 \operatorname{deg}
$$

Finally, in order to account for the actuator time delay of $0.2 \mathrm{~s}$, a lead compensator $L(s)$ is designed given by $L(s)=\frac{8(s+4.5)}{4.5(s+8)}$. Furthermore, a derivative filter of the form $D(s)=\frac{12(s+4)}{s+8}$ is designed. Finally, the commanded dihedral deflection is given by

$$
\delta_{a}=k_{d} D(s) L(s) e_{r}(s)
$$

\section{Guidance Loop}

The outer control loop is designed to ensure rapid changes in the flight path over a short duration. For the sake of completeness, it must be noted here that, in general, the guidance loop commands the flight path angle as well as the turn rate. The flight path angle $(\gamma)$, the heading angle $(\chi)$, and the turn rate $(\omega)$ are given $b^{3}$

$$
\begin{aligned}
& \sin \gamma=\cos \alpha \cos \beta \sin \theta-\sin \beta \sin \phi \cos \theta-\sin \alpha \cos \beta \cos \phi \cos \theta \\
& \sin \chi \cos \gamma=\cos \alpha \cos \beta \cos \theta \sin \psi+\sin \beta(\sin \phi \sin \theta \sin \psi+\cos \phi \cos \psi) \\
& +\sin \alpha \cos \beta(\cos \phi \sin \theta \sin \psi \sin \phi \cos \psi) \\
& \omega=\dot{\chi}=\operatorname{sign}(\dot{\chi}) \sqrt{p^{2}+q^{2}+r^{2}}
\end{aligned}
$$

The flight path angle is controlled in discrete time so that a symmetric dihedral angle is commanded every $0.2 \mathrm{~s}$ (which is equal to the dihedral acutator time delay). The commanded dihedral angles are given by

$$
\delta_{R}=\delta_{L}=\sqrt{2+\frac{2}{\zeta(\alpha) \tan \gamma_{c}}}, \quad \zeta(\alpha) \approx \frac{C_{L}(\alpha)}{C_{D}(\alpha)}
$$

where $\gamma_{c}$ is the commanded flight path angle which is, in turn, given by

$$
\gamma_{c}=\frac{h}{1+0.28125 h^{2}}, \quad h=\frac{z-z_{l}}{\sqrt{\left(x-x_{l}\right)^{2}+\left(y-y_{l}\right)^{2}}}
$$

Here, $x_{l}, y_{l}$ and $z_{l}$ are the coordinates of the desired landing point on the ground, or a point in the air where a perching command is to be sent to the aircraft. It has to be noted that the dihedral and flight path angles are computed together every $0.2 \mathrm{~s}$. This is not an optimal gliding strategy because it does not take 
into account the instantaneous flight path angle and aircraft speed. It was seen to be effective over the short duration of the experiments, although it needs to be improved for experiments which may last over a longer duration. It is interesting to note that changing the wing dihedral brings about a significant effect in the pitching moment and using a continuous-time flight path controller leads to undesirable oscillatory behavior due to coupling with the pitch dynamics.

\section{Experimental Setup}

The design of the MAV has been described in detail in this section, along with details of the experimental setup.

\section{A. Articulated MAV Design}
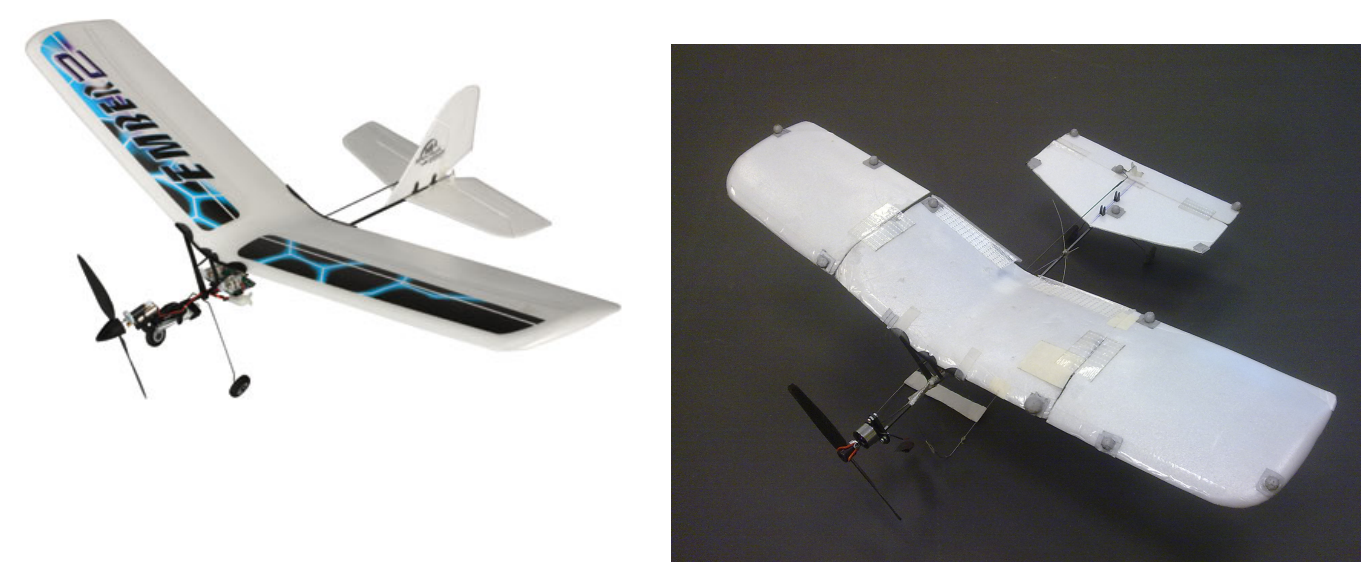

(a) The ParkZone Ember 2 RTF

(b) Tailless articulated MAV with additional actuators

Figure 5. ParkZone Ember 2 in Original Manufactured Form and the Redesigned Tailless Articulated MAV

For the development of a test MAV, the commerically manufactured ParkZone Ember 2, shown in Fig. 5, was redesigned. ${ }^{13}$ Carbon fiber rods were used for the airplane structure, and the aerodynamic surfaces were made of depron foam. The original wing was cut to facilitate hinging of the outboard $60 \%$ of the wing. Extremely lightweight hinges were made from fiberglass-reinforced tape. Actuators were attached on the lower surface of the center (non-rotating) wing section, along with the radio receiver. The original vertical tail was removed, and a resized horizontal tail was installed. A $3.7 \mathrm{~V}, 125 \mathrm{mAh}$ battery powers a $2.4 \mathrm{GHz}$ receiver which controls actuators for wing dihedral and the elevator, as well as the electric motor. Due to the increased weight and likelihood of damage during testing, a spring-steel cage was outfitted to protect the wing and motor assembly. The increase in weight also prompted the replacement of the motor with a high-performance brushless outrunner system. The newly-designed MAV exceeded the original weight by 27 grams. The geometric properties for the MAV are listed in Table 1.

Both wings are free to rotate from a maximum 45 deg dihedral to minimum -15 deg anhedral for a total arc range of 60 degrees. The actuators for wing dihedral, it may be recalled, are controlled independently on both wings for yaw stability and control. ${ }^{3}$ Bourdin, Gatto and Friswell ${ }^{14}$ introduced a similar idea for a larger aircraft operating at high Reynolds numbers, whereas this experiment is focused on MAVs operating at a low Reynolds number. Various configurations using the asymmetric dihedral wings are shown in Figure 6. Digital actuators with a torque rating of $0.29 \mathrm{~kg}-\mathrm{cm}^{15}$ are powerful enough to maneuver the wings. The time required for the wings to rotate from the minimum $-15 \mathrm{deg}$ to maximum $45 \mathrm{deg}$ is about $0.05 \mathrm{~s}$, which 
Table 1. Physical Properties of the MAV

\begin{tabular}{|l|c|c|}
\hline Property & Metric Measurement & Units \\
\hline \hline Mass & 44.0 & $\mathrm{~g}$ \\
\hline Wing span & 41.8 & $\mathrm{~cm}$ \\
\hline Wing chord (at root) & 9.5 & $\mathrm{~cm}$ \\
\hline Wing incidence angle & 6.0 & $\mathrm{deg}$ \\
\hline Wing dihedral & controlled-variable & \\
\hline MAV length & 35.0 & $\mathrm{~cm}$ \\
\hline Elevator area & 39.12 & $\mathrm{~cm}^{2}$ \\
\hline Propeller Thrust & 39 & $\mathrm{~g}$ \\
\hline
\end{tabular}

is quick enough for a rapid perching manuever.

\section{B. VICON System}

The VICON motion-capture system is comprised of 16 infrared cameras, each with its own IR light source. Reflective markers are attached on the various articulated parts of the MAV, while cameras track the marker reflections in their field of view. Using the method of triangulation, the VICON system is able to locate the markers accurately in all three dimensions. A recording rate of $100 \mathrm{~Hz}$ is used to capture the position and orientation data.

The MAV is modeled in the VICON software as a collection of multiple rigid objects so that each articulated part can be independently tracked. For each component, the earth reference position and the Euler angles are recorded. Figure 7 shows the MAV objects constructed in the VICON software. Tracking data is used for real-time, closed-loop experiments as well as post-processing to determine the aircraft's performance throughout the flight duration.

\section{Data Acquisition}

The real-time datastream provided by the VICON motion-capture system includes the global reference position and the Euler angles of each object. The availability of tracking data is contingent upon the visibility of the objects. For time-steps with information loss, which were minimal and rarely comprised consecutive frames, a linear fit is used to estimate the missing data. Experiments are performed within the effective volume of capture, containing an area of $6 \mathrm{~m} . \times 4 \mathrm{~m}$. and a height of $2 \mathrm{~m}$.. Since VICON provides only position and attitude information, a second order Lagrangian polynomial is used to compute velocities and angular rates, which are then filtered to eliminate noise. Using rotation matrices of the actuated wings, dihedral angles are determined with respect to the fuselage. Figure 8 shows a VICON view of the wings, each with its own body-axis frame. The VICON software development kit is interfaced with MATLAB to calculate aircraft flight parameters for performance assessment and closed-loop feedback control. As VICON transmits data to MATLAB, a real-time controller commands a new set of values to the control surfaces, including the dihedral actuators on the wings. Control command signals are radioed back to the on-board receiver.

\section{Experiments}

\section{A. Angle of Attack Control}

Figure 9 shows the experimentally-measured longitudinal flight parameters. For these experiments, the wing dihedral was not controlled actively which caused the aircraft heading to deviate steadily. An angle of attack of $5 \mathrm{deg}$ was commanded while the flight speed and flight path angle were not controlled. Figure 10 plots the same parameters for a similar experiment performed to verify that similar stability characteristics as the first experiment could be reproduced. Figure 10 also shows the angular rates, sideslip and the velocity heading angle, all of which are stabilized by the vertical tail. The controller for Configuration B yielded similar characteristics as Configuration A. 


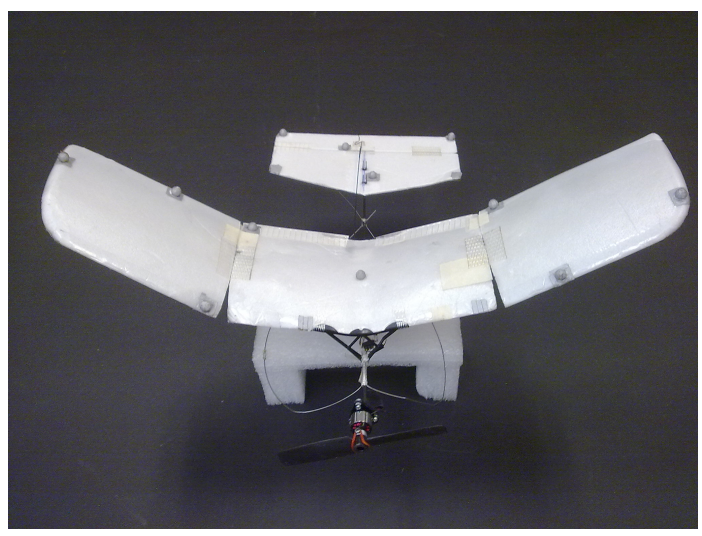

(a) Symmetric dihedral 40 degrees

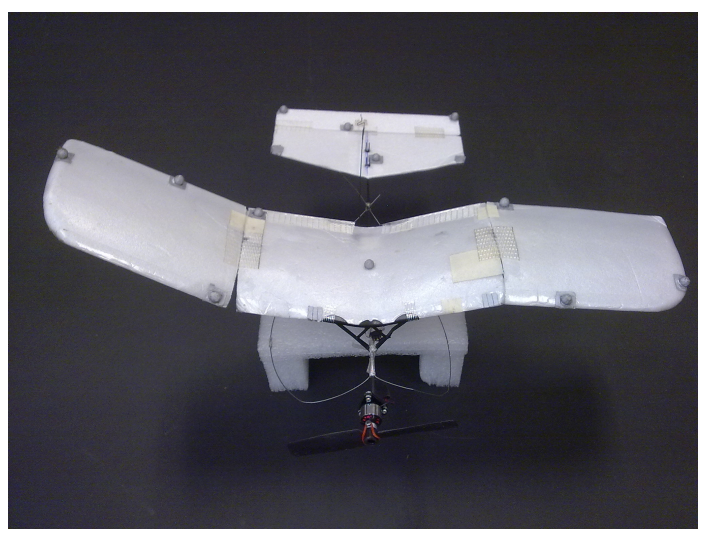

(c) Asymmetry with elevated right wing

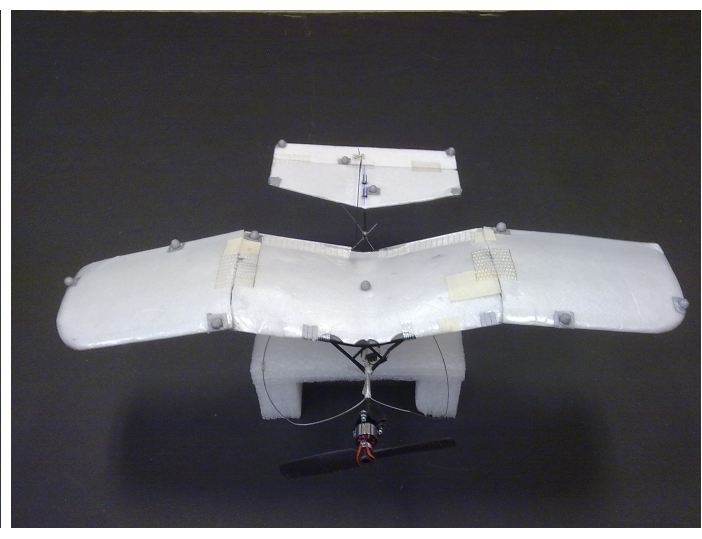

(b) Symmetric anhedral of -15 degrees

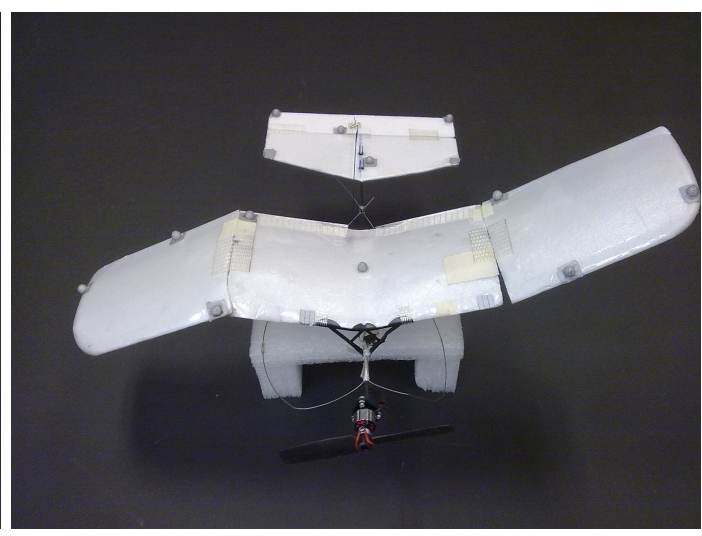

(d) Asymmetry with elevated left wing

Figure 6. Various Configurations of the Asymmetric Dihedral Wings. The foam table on which the aircraft is resting is not part of the airframe.

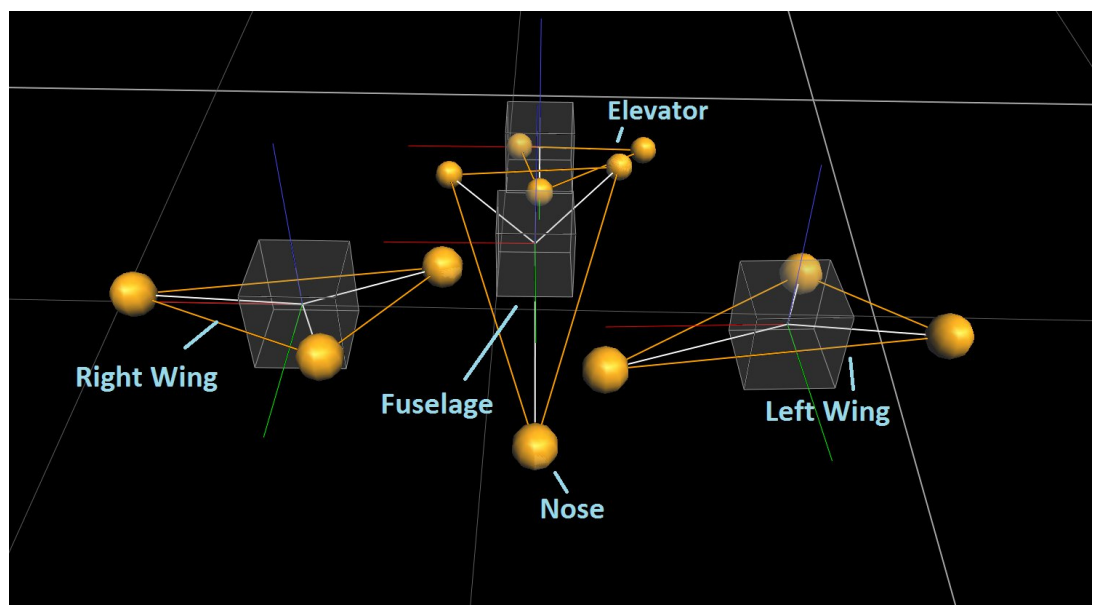

Figure 7. Reflective markers are grouped into separate objects with the fuselage, right wing, left wing, and the elevator being tracked in the VICON software 


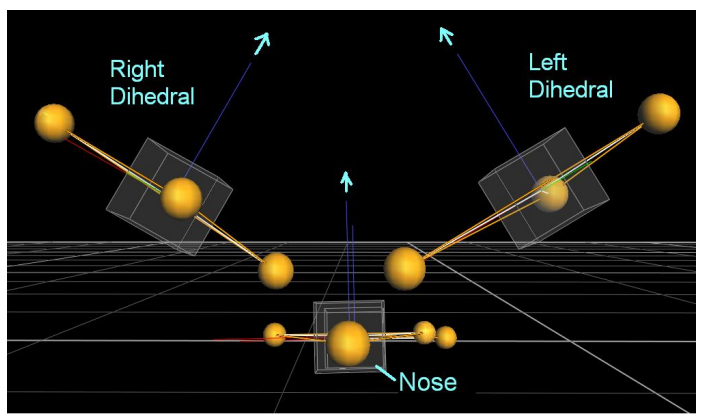

Figure 8. Dihedral angles and lift vector determined through the VICON system

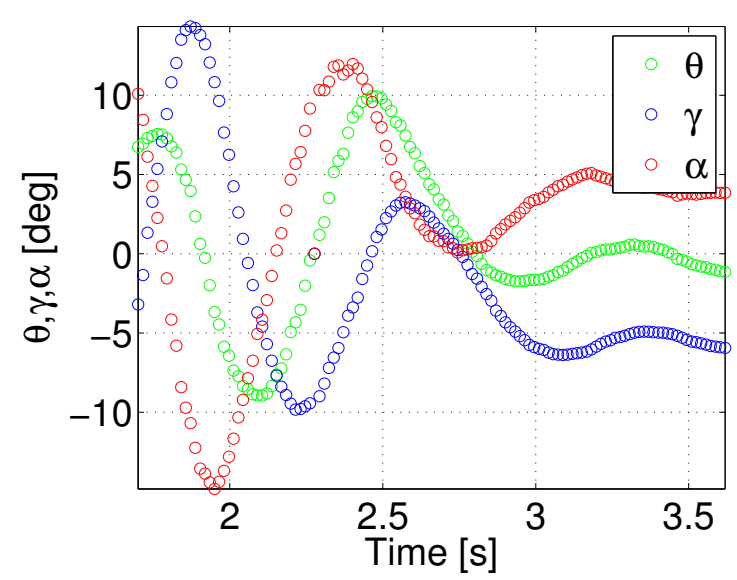

(a) $\alpha, \theta$ and $\gamma$

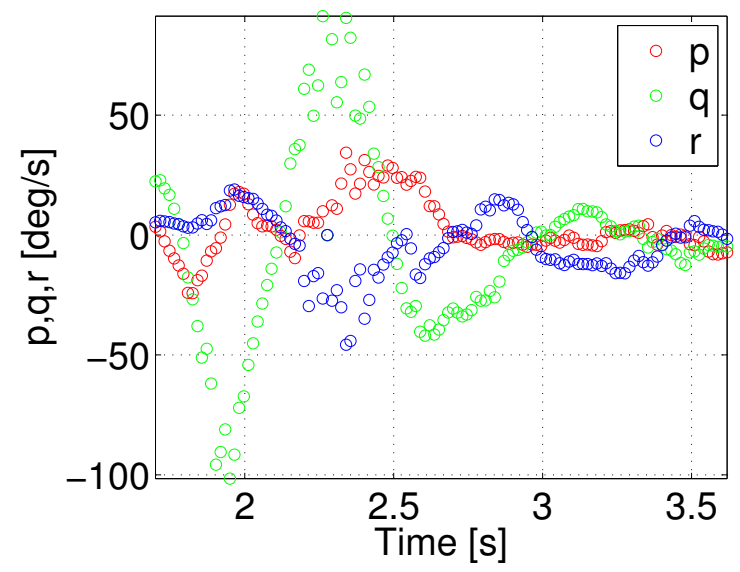

(c) Angular rates

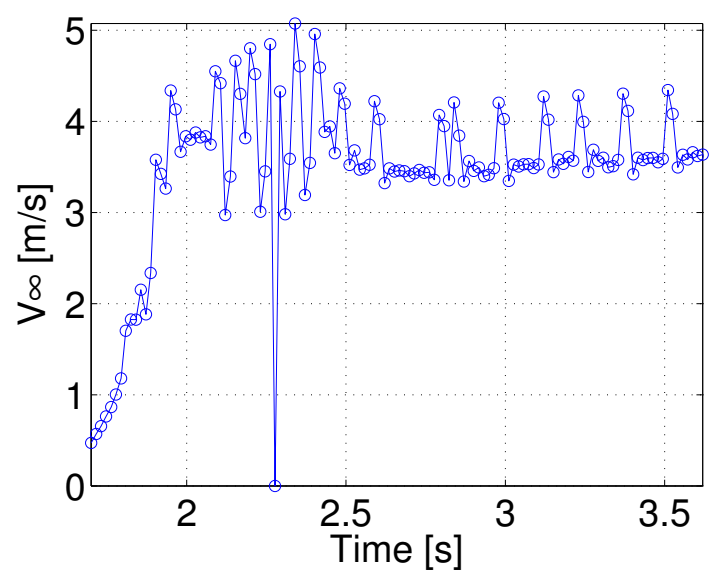

(b) Flight speed
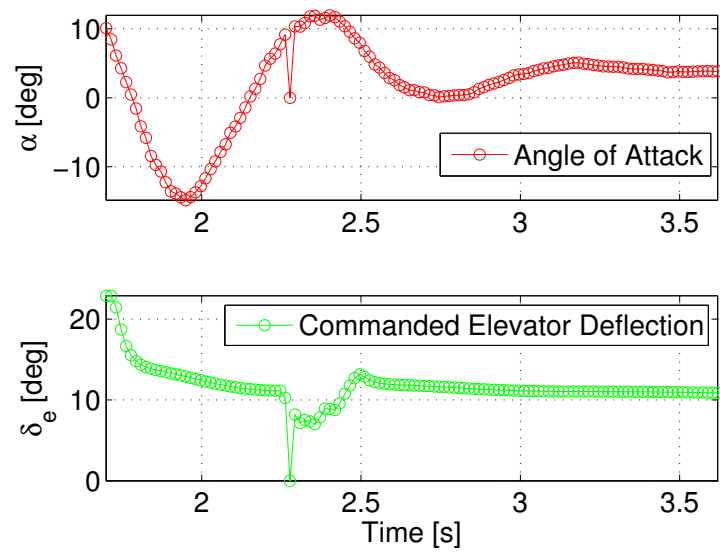

(d) Elevator Deflection

Figure 9. Experimental results showing the longitudinal flight parameters. In particular, $\alpha$ settles down at the desired value within $2 \mathrm{~s}$. The outlying peaks around $2.2 \mathrm{~s}$ are due to a lost data packet. They do not affect the performance of the controller. 
Pitch, Filtered Flight Path, Attack Angles [deg]

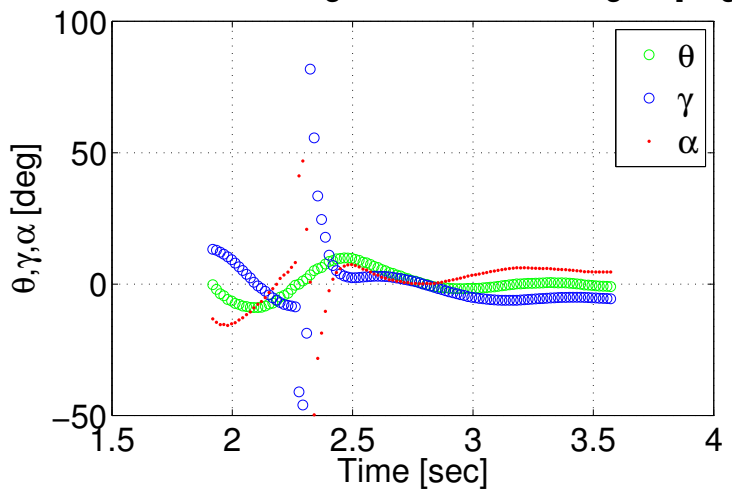

(a) $\alpha, \theta$ and $\gamma$

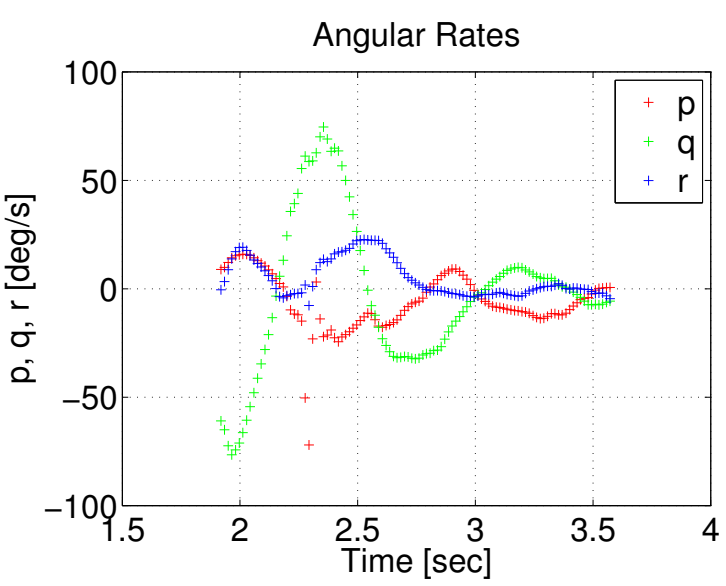

(c) Angular rates

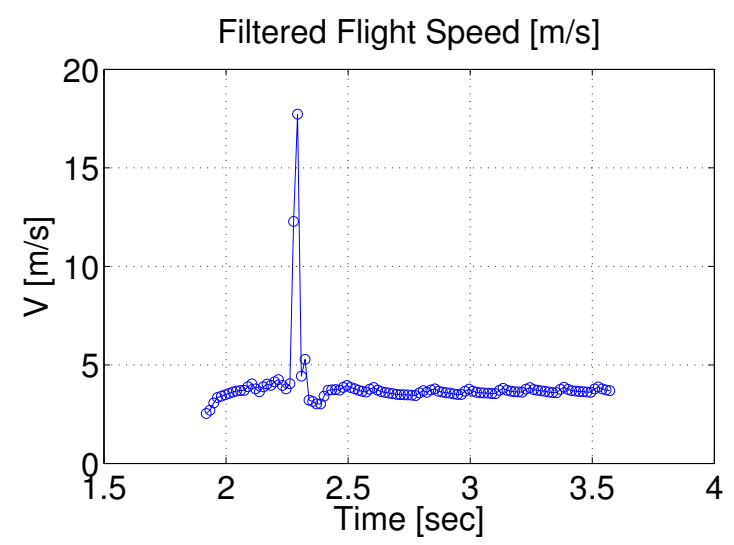

(b) Flight speed

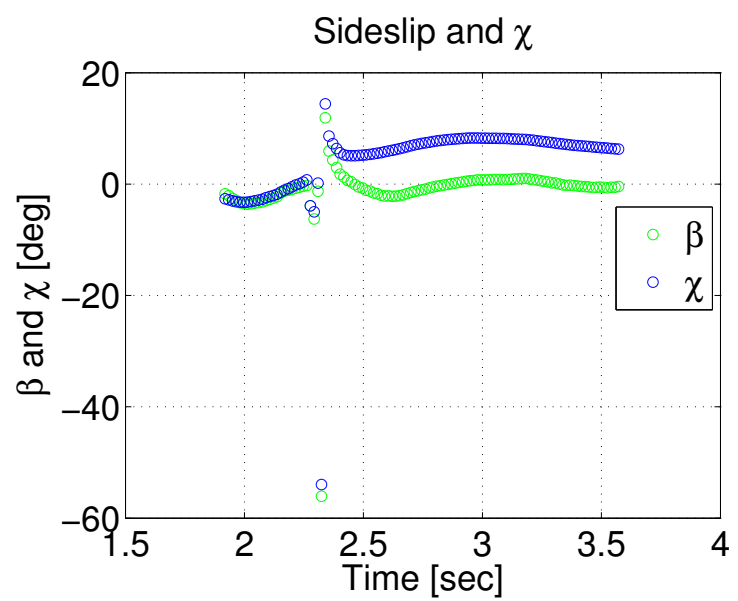

(d) Sideslip and velocity heading

Figure 10. Experimental results showing the longitudinal flight parameters for Configuration A. In particular, $\alpha$ settles down at the desired value within $2 \mathrm{~s}$. The peak around $2.5 \mathrm{~s}$ is due to a lost data packet. Although it appears on the plot, it is not fed to the controller. 


\section{B. Lateral Control}

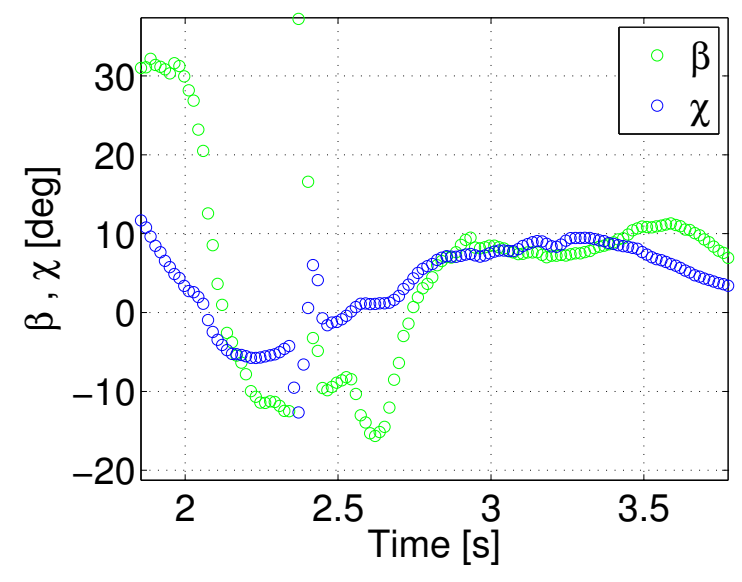

(a) Sideslip and velocity heading

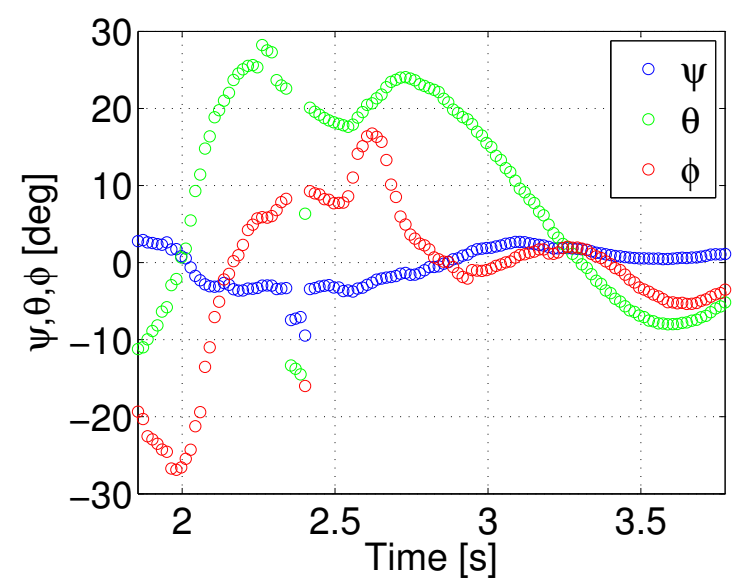

(c) Euler angles

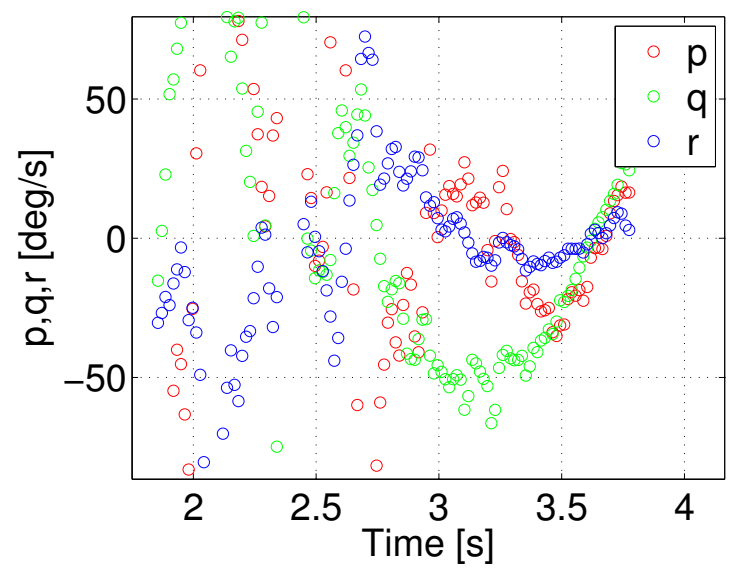

(b) Angular rates
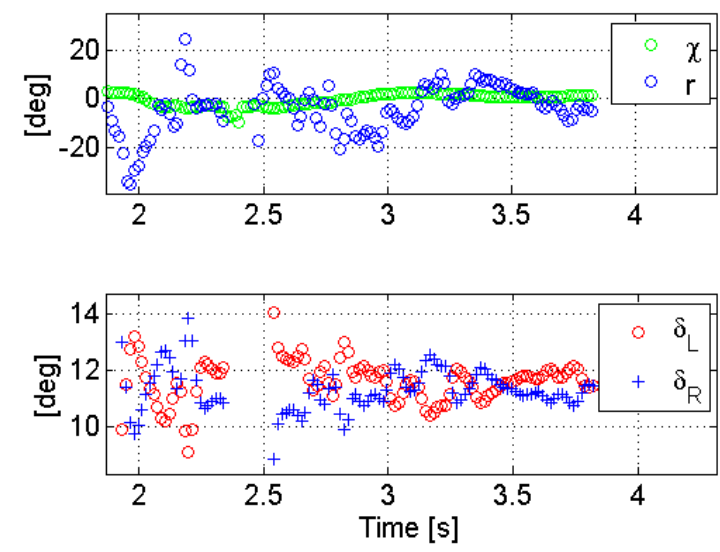

(d) Control inputs vs. outputs

Figure 11. Experimental results showing various flight parameters during yaw control testing of Configuration A. Parameters appear to be regulating during the short experiment

In Configuration A, local lateral stability was achieved using a simple PID controller. However, in several flight tests, the roll rate was seen to build up due to the dihedral effect and, without wing twist or ailerons, could not be compensated. This led to a divergent lateral-directional behavior despite local stability. Figure 11 shows the time histories for the case where the lateral dynamics were seen to be stable. A zero heading angle was commanded. The heading angle as well as sideslip converge to small values. However, the transient response does not vanish within the limited flight duration. Nevertheless, the yaw rate slows significantly by the end of the flight indicating good closed loop stability characteristics.

Lateral control of Configuration B is under experimental investigation. Preliminary results are presented in Figs. 12 for different combinations of $\delta_{e}$ and $k_{D}$. The yaw rate shows a tendency to settle down, although the dynamics are not fast enough for the yaw rate to converge within the duration of the experiment.

\section{Flight Path Control}

An effective flight path controller is necessary for a successful perching maneuver. The aircraft must be able to track the desired flight path in order to arrive at a spatial target with an acceptable flight speed and 


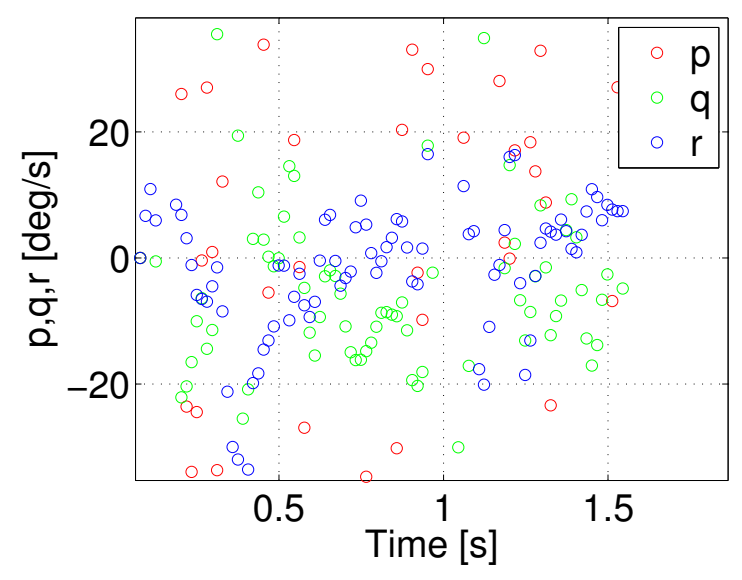

(a) $k_{d}=2.5, \delta_{e}=0$

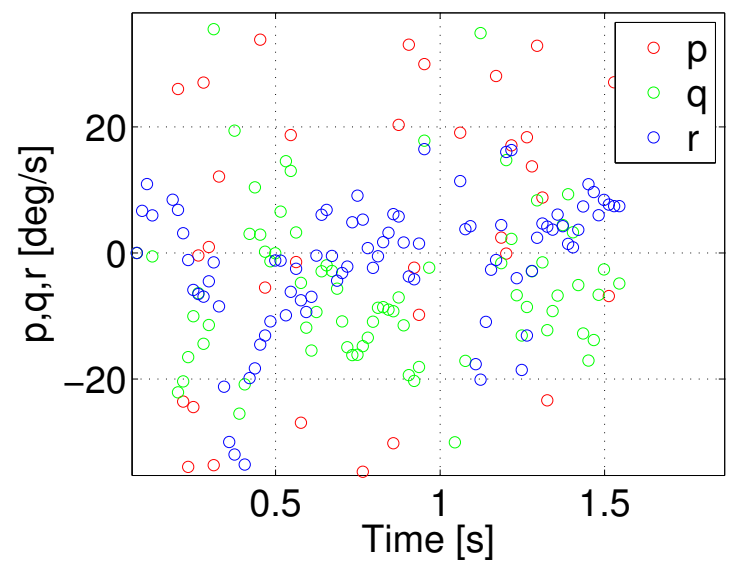

(b) $k_{d}=3.5, \delta_{e}=-5 \mathrm{deg}$

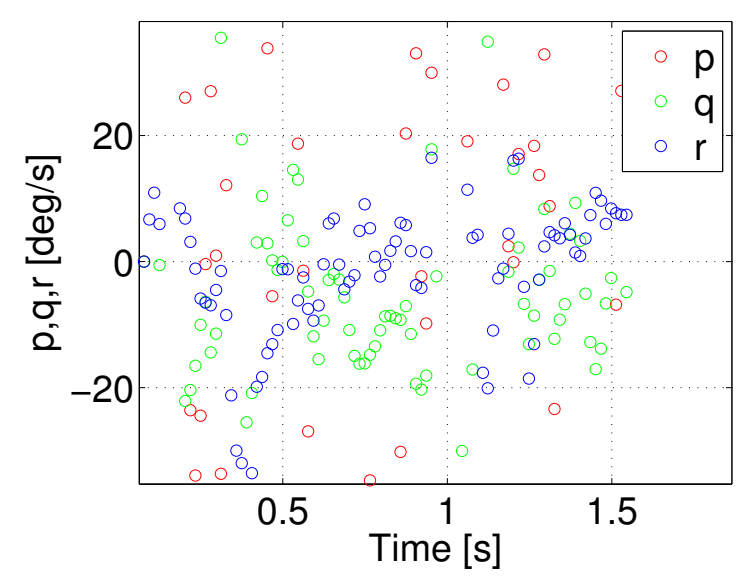

(c) $k_{d}=3.5, \delta_{e}=0$

Figure 12. Experimental results showing various flight parameters during yaw control testing of Configuration B. The yaw rate appears to be converging, although the relatively large value of the roll rate is an impediment. 
height. The PID controller gains were tuned to provide consistent tracking across a range of flight path angles. Experiments were first conducted to demonstrate simultaneous tracking of flight path angle and angle of attack. Figure 13 shows two experiments where flight path angle and angle of attack succesfully follow the command. Dynamic flight path angle commands used here are necessary to accurately arrive at the prescribed location.

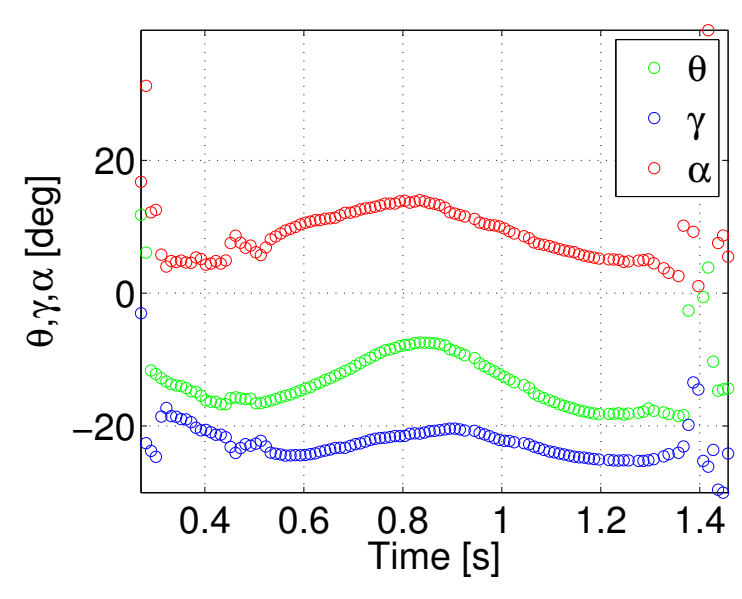

(a) $\gamma_{\text {command }}=-20 \mathrm{deg}$

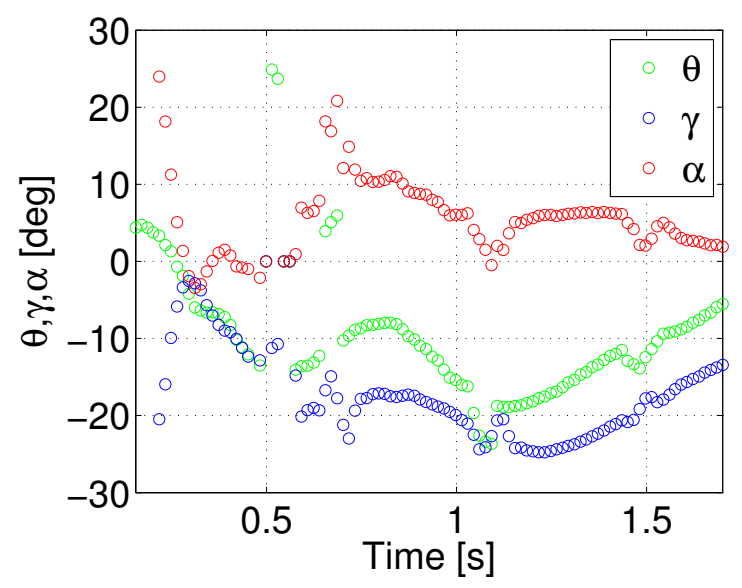

(b) $\gamma_{\text {command }}=-15 \mathrm{deg}$

Figure 13. Angle of attack and flight path angle during flight path guidance control, where angle of attack is commanded to $-10 \mathrm{deg}$ in each trial

\section{Perching Maneuver}

In conjunction with the guidance controller, a perching maneuver is executed as follows. An appropriate altitude is chosen such that a perching command is sent when the aircraft crosses it. This value was chosen to accommodate the actuation time delays for the wing dihedral as well as the elevator. Once the aircraft reaches the prescribed altitude, zero dihedral and maximum pitch-up elevator angles are commanded. These signals are held until touch-down. Figure 14 shows the perching signal sent at the $0.6 \mathrm{~s}$ mark. The angle of attack builds up to $30 \mathrm{deg}$, causing the speed to reduce, and the aircraft climbs momentarily. Flight speed is halved within $1 \mathrm{~s}$ to $3 \mathrm{~m} / \mathrm{s}$. After a brief ascent, the MAV lands at a low angle of attack. It is interesting to note that the final speed has reduced substantially even without using wing twist. Addition of wing twist would not only enable a further reduction in the final speed, but also provide for better roll and yaw control during the approach. Figure 15 shows a perching trajectory recorded by Vicon.

\section{E. Limitations of the Experimental Setup}

One of the limitations in the MAV is the time lag in the actuator response. Figure 16 shows the actual and the commanded time histories of the dihedral angles and the elevator. It is evident that the actual response of the dihedral wings and the elevator lags the commanded values by approximately $0.2 \mathrm{~s}$. Furthermore, the digital filters implemented to compensate for the time delay amplify noise in the output and are designed with a low order Padé approximation. Due to torque limitations of the servos and their limited ability to handle high wing-loading, the dihedral angles are typically $10-15$ deg higher than the commanded values.

\section{Conclusions}

This paper described perching experiments using a novel MAV concept featuring independent wing dihedral actuation for longitudinal as well as yaw control. A guidance and control scheme was designed for the MAV and closed loop experiments were performed indoors to demonstrate its perching capability. Preliminary results indicate sound yaw control characteristics. Future work will focus on improving the lateral-directional control capability of the wing dihedral mechanism and adding heading tracking capability. 


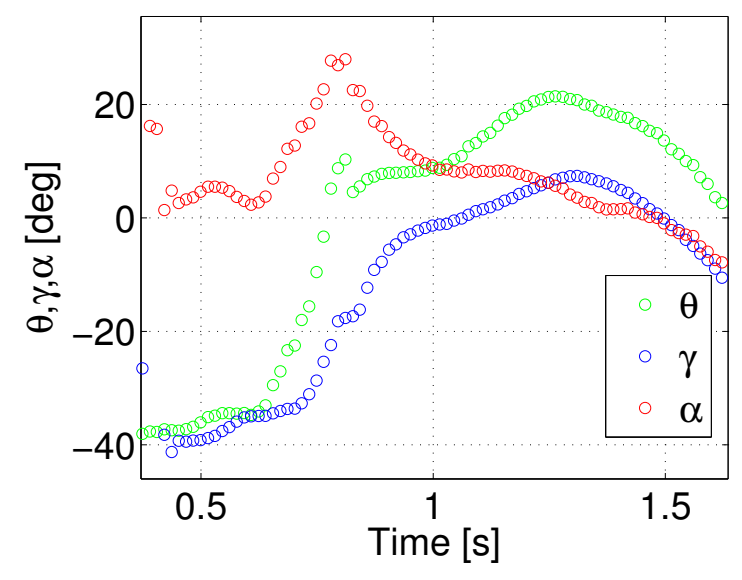

(a) $\alpha, \theta$ and $\gamma$

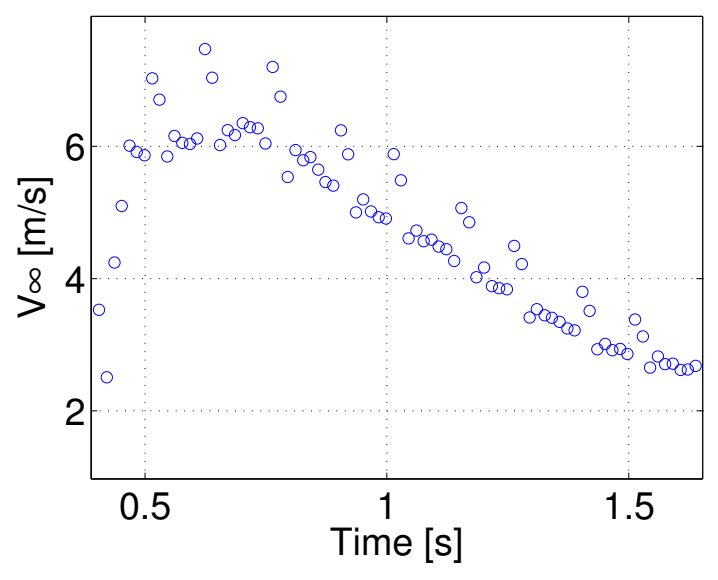

(b) Flight Speed

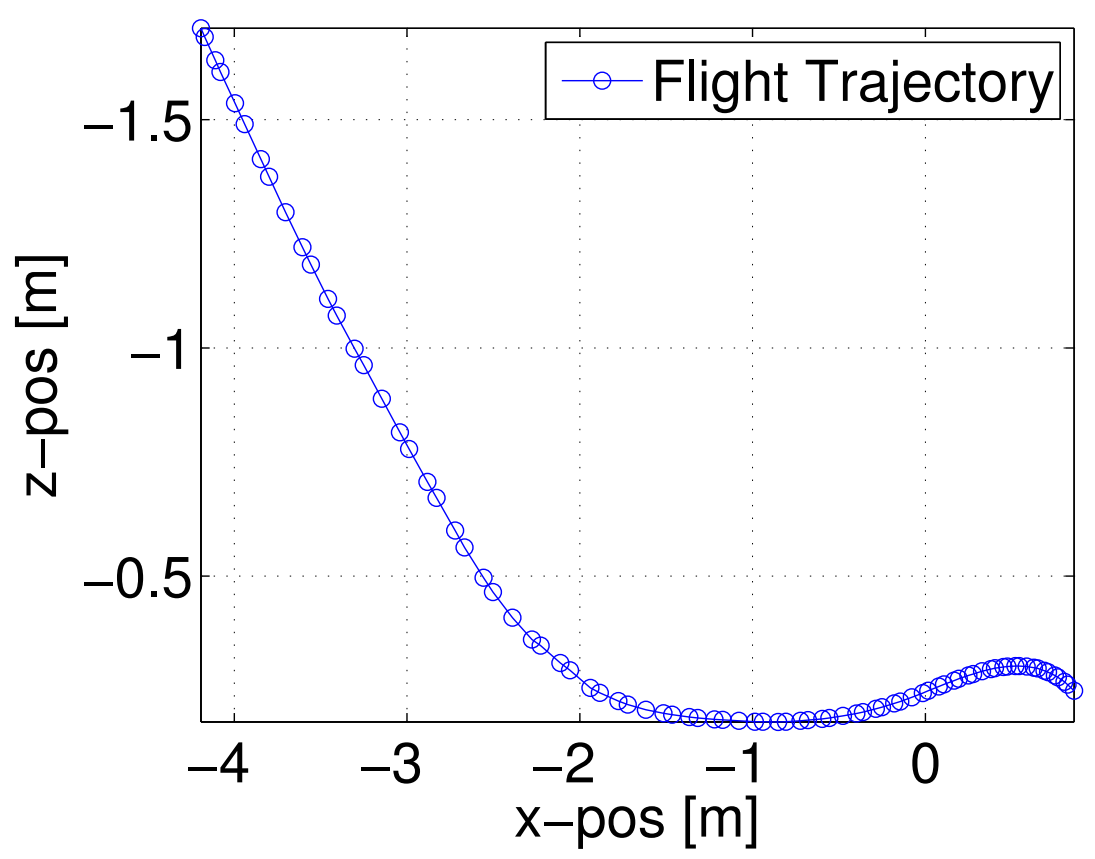

(c) Trajectory

Figure 14. Flight parameters during a perch attempt that was triggered at $1.5 \mathrm{~m}$ above the ground 


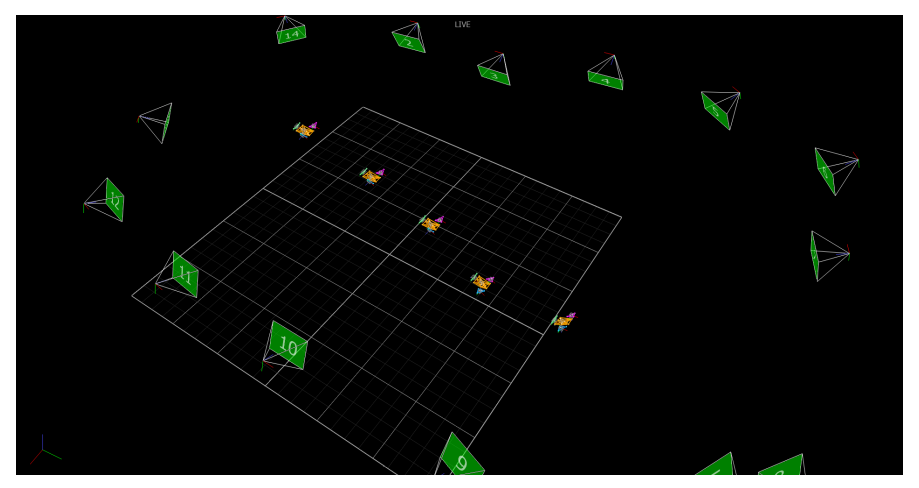

(a) Top view

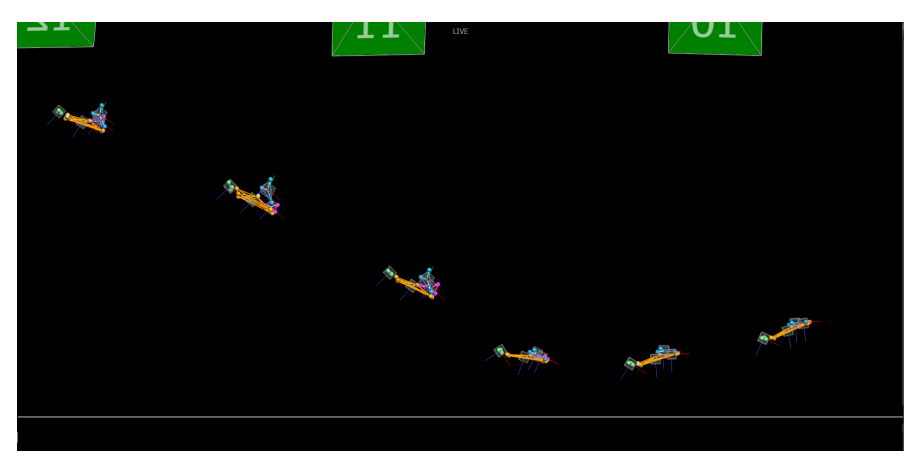

(b) Side view

Figure 15. Perching trajectory, as seen by the Vicon cameras.

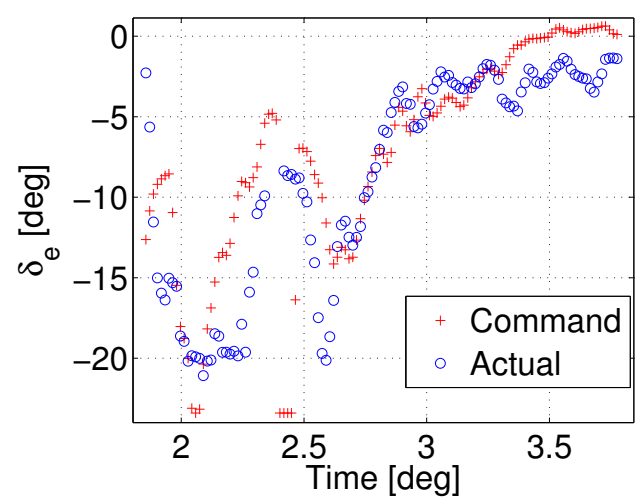

Figure 16. Elevator deflection showing time delay of 0.2 seconds 


\section{Acknowledgment}

This project was supported by the Air Force Office of Scientific Research (AFOSR) under the Young Investigator Award Program (Grant No. FA95500910089) monitored by Dr. W. Larkin. The original problem was posed by Dr. Gregg Abate (AFRL). This paper also benefitted from stimulating discussions with Mr. Johnny Evers (AFRL) and Dr. Animesh Chakravarthy. The authors thank undergraduate research assistants Dan Chuchawat, Li Pan, Michael Looby, and Daniel Park for their contributions to the experiments, and Prof. James Phillips (MechSE UIUC) and Mr. Greg Milner (AE UIUC) for allowing us to schedule experiments quite liberally in an otherwise busy materials testing facility.

\section{References}

${ }^{1}$ Mueller T. J. 2001 Fixed and Flapping Wing Aerodynamics for Micro Air Vehicle Applications Progress in Aeronautics and Astronautics, 195, American Institute of Aeronautics and Astronautics (AIAA) Reston, VA.

${ }^{2}$ Sanders, B., Crowe, R., and Garcia, E., "Defense Advanced Research Projects Agency: Smart Materials and Structures Demonstration Program Overview," Journal of Intelligent Material Systems and Structures, Vol. 15, No. 4, 2004 , pp. $227-223$.

${ }^{3}$ Paranjape, A. A., Chung, S.-J., and Selig, M. S., "Flight Mechanics of a Tailless Articulated Wing Aircraft," Bioinspiration ES Biomimetics, Vol. 6, No. 2, 2011.

${ }^{4}$ Wickenheiser, A., Garcia, E., and Waszak, M., "Longitudinal Dynamics of a Perching Aircraft Concept," Proceedings of SPIE: International Society of Optical Engineering, Vol. 5764, May 2005, pp. 192-202.

${ }^{5}$ Wickenheiser, A., and Garcia, E., "Longitudinal Dynamics of a Perching Aircraft," Journal of Aircraft, Vol. 43, No. 5, 2006, pp. 1386-1392.

${ }^{6}$ Crowther, W. J., "Perched Landing and Takeoff for Fixed Wing UAVs," NATO Symposium on Unmanned Vehicles for Aerial, Ground, and Naval Military Operations, 2000.

${ }^{7}$ Wickenheiser, A., and Garcia, E., "Optimization of Perching Maneuvers Through Vehicle Morphing," Journal of Guidance, Control, and Dynamics, Vol. 31, No. 4, 2008, pp. 815-823.

${ }^{8}$ Reich, G. W., Wojnar, O., and Albertani, R., "Aerodynamic Performance of a Notional Perching MAV Design," AIAA Paper 2009-63, 2009.

${ }^{9}$ Roberts, J. W., Cory, R., and Tedrake, R., "On the Controllability of Fixed-Wing Perching," Proc. American Control Conference, St. Louis, MO, June 10-12 2009, pp. 2018 - 2023.

${ }^{10}$ Desbiens, A. L., and Cutkosky, M. R., "Landing and Perching on Vertical Surfaces with Microspines for Small Unmanned Air Vehicles," Journal of Intelligent Robotic Systems, Vol. 57, 2010, pp. 313-327.

${ }^{11}$ Cory, R. and Tedrake, R., "Experiments in Fixed-Wing UAV Perching," AIAA Paper 2008-7256, 2008.

${ }^{12}$ Bethke, B., Valenti, M., and How, J. P., "UAV Task Assignment," IEEE Robotics and Automation Magazine, Vol. 15, No. 1, 2008, pp. 39-44.

${ }^{13}$ ParkZone, "Ember 2 RTF," http://www.advantagehobby.com/popup_image.php?type=D\&id=103913\&title =Ember\%202\%20RTF\&area=C, Accessed January 2011.

${ }^{14}$ Bourdin P. Gatto A. and Friswell M., 2008, "Aircraft Control via Variable Cant-Angle Winglets," Journal of Aircraft, Vol. 45, No. 2, 2008, pp. 414-423.

${ }^{15}$ Blue Arrow, "3.6 Gram Micro Servo," http://www.rctoys.com/rc-toys-and-parts/BA-36G/ RC-PARTS-BLUE-ARROWSERVOS.html, Accessed January 2011.

${ }^{16}$ Johnson, E. N., Turbe M. A., Wu, A. D., Kannan S. K., and Neidhoefer, J. C., "Flight Test Results of Autonomous Fixed-Wing UAV Transitions to and from Stationary Hover," AIAA Paper 2006-6775, 2006.

${ }^{17}$ Klein, V. and Morelli, E. A., Aircraft System Identification: Theory and Practice, AIAA Education Series, AIAA, Reston, VA, 2006

${ }^{18}$ Jategaonkar, R. V., Flight Vehicle System Identification: A Time Domain Methodology, AIAA Progress in Astronautics and Aeronautics, AIAA, Reston, VA, 2006

${ }^{19}$ Wang, Q., and Stengel, R. F., "Robust Nonlinear Flight Control of a High-Performance Aircaft," IEEE Trans. Control Systems Technology, Vol. 13, No. 1, 2005, pp. $15-26$. 